\title{
Wideband High-Gain Directly Coupled Circularly Polarized Microstrip Patch Antenna with Shorting Pin
}

\author{
T. Biradar ${ }^{1}$ and K. Reddy ${ }^{2}$ \\ ${ }^{1}$ Department of Electronics and Telecommunication, Rajiv Gandhi Institute of Technology, Versova, Mumbai, \\ Maharashtra, India-400053 \\ ${ }^{2}$ Pranveer Singh Institute of Technology, Kanpur, UP-209305. \\ \{tanajib@rediffmail.com; ktvreddy@gmail.com\}
}

\begin{abstract}
A wideband circularly polarized microstrip patch (CP MPA) antenna directly coupled with single feed structure is proposed for wide axial ratio bandwidth (AR BW). The antenna is basically designed for circular polarization and is composed of suspended near square patch with diagonal single feed. The near square patch is directly coupled with two neighbour parasitic patches. By loading a shorting pin in the diagonal of the parasitic patch, wide AR BW and high gain is obtained. The design is carried out at the $3 \mathrm{Ghz}$ as centre frequency. The proposed antenna is fabricated and measured for radiation patterns. The square patch with two nearly square parasitic patches has been directly coupled. This configuration has a simulated and measured, 10$\mathrm{dB}$ return-loss bandwidth (RL BW) of $13.3 \%$ and $16.06 \%$ respectively. This configuration yields simulated $3 \mathrm{~dB}$ AR BW of $6.79 \%$, with an average gain of $8.5 \mathrm{dBi}$ across the AR frequency range with the above defined centre frequency.
\end{abstract}

Keywords: Square patch antenna, circular polarization, shorting pin, suspended and directly-coupled antenna.

\section{Introduction}

Circularly Polarized mictrostrip Antennas (CP MSA) are used for greater flexibility in orientation between transmitters and receivers compared to the linear polarization for reduction in multipath reflections, weather penetration and better mobility. Therefore, CP antennas have become wide area of interest for mobile, radar and satellite communications etc. [2]. A CP MSA can be realized by exciting two orthogonal modes with equal magnitudes, which are in phase quadrature and which can be achieved by using single feed or multi feed. The single feed CP MSA is always preferred because of simple a structure that does not require an external polarizer over dual feed, therefore such antenna can be realized more compactly by using less board than dual feed CP MSAs. Low gain and narrow bandwidth are major disadvantages associated with microstrip antennas. To broaden the axial ratio (AR) bandwidth of CP antennas various techniques are used, some of these techniques include slot cut structures, multiresonator and use of thicker substrate with low permittivity [2][8]. Some of the techniques used to realize CP are square or equilateral triangle MSAs, diagonally feed square MSA, chopping diagonal corners of the rectangular microstrip antenna (RMSA) and cutting slot inside circular [5].

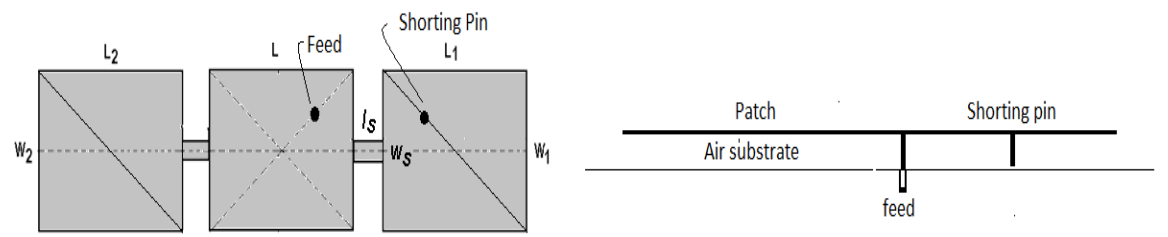

Fig. 1. Configuration of proposed antenna.

In [1], it has been shown that the VSWR bandwidth of microstrip patch antennas enhanced by adding two additional resonators, which are directly coupled to the radiating edges of a RMSA.

B. Iyer, S. Nalbalwar and R. Pawade (Eds.)

ICCASP/ICMMD-2016. Advances in Intelligent Systems Research.

Vol. 137, Pp. 565-569.

(C) 2017- The authors. Published by Atlantis Press

This is an open access article under the CC BY-NC license (http://creativecommons.org/licens)es/by-nc/4) 
Reference [4][3] describes the design of circularly polarized planar antennas for a cross array of five patches and a square array of nine elements which is fed by two probes. The cross-array gives axial ratios over a $3.3 \%$ bandwidth, and wide angular range, but square array of nine elements gives lesser axial ratio bandwidth. In [9], to obtain wide AR BW, circularly polarized antenna with L-shaped strip feeding and shorting-pin is proposed and AR BW of $17.9 \%$ is achieved. By using chip resistor techniques in place of shorting posts, AR bandwidth is broadened at the expense of gain [7]. In [6], CP is achieved by placing shorting post on the radiating patch near the edges of diagonal-fed patch.

In this paper, we present a suspended near square patch with single feed and loaded the parasitic patch with shorting pin configuration to achieve wide AR BW and large gain. For tuning resonate mode easily and for large $\mathrm{CP}$ bandwidth shorting pin is loaded in the diagonal of parasitic patch. The organization of the paper is as follows. The Section II is concerned with the proposed antenna configuration. The Section III discusses the simulated results. Finally, Section IV concludes the work.

\section{Antenna Design and Analysis}

Configuration of proposed antenna is shown in Fig. 1. The antenna consist of a suspended near square patch printed on an FR4 substrate (relative permittivity $=4.4, \mathrm{~h}=1.6 \mathrm{~mm}$, loss tangent $=0.02$ and radii of probe $0.6 \mathrm{~mm}$ ), diagonal feed, shorting pin at diagonal of parasitic patch. This shorting pin is actually a feed attaching parasitic patch to ground plane. Two near square parasitic patches are directly-coupled using thin resonant length of transmission line to the central radiating patch. Air substrate is used in this configuration to achieve wide bandwidth, larger gain and lower cost. The configurations in later sections are optimized and simulated with IE3D software.

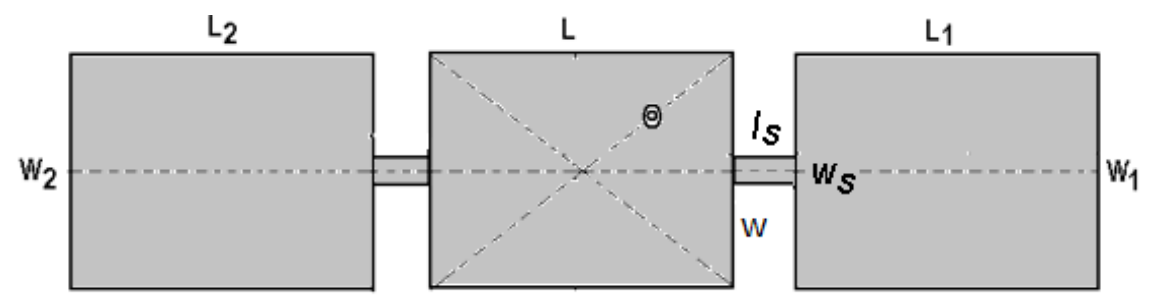

Fig. 2. Two patches directly-coupled microstrip patch antenna $[\mathrm{L}=33.8 \mathrm{~mm}, \mathrm{~W}=31.3 \mathrm{~mm}, \mathrm{~L} 1=32.8 \mathrm{~mm}, \mathrm{~L} 2=32.2 \mathrm{~mm}$, $\mathrm{W} 1=\mathrm{W} 2=31.3 \mathrm{~mm}$ ls $1=1 \mathrm{~s} 2=8.8 \mathrm{~mm}, \mathrm{Ws}=3 \mathrm{~mm}$, hair $=1.6 \mathrm{~mm}]$

\subsection{Antenna without shorting pin}

We first investigate the performance of proposed configuration without shorting pin. By changing various dimension of antenna i.e. probe feeding position, length and width of the patch, two orthogonal modes can be excited which are equal amplitudes and a $90^{\circ}$ phase difference, which results in CP operation at a frequency. Center rectangular patch is designed such that it resonates at $3 \mathrm{GHz}$ and is found to be $33.8 \mathrm{X} 31.3 \mathrm{~mm}$. By adjusting feed point position on the diagonal of radiating patch, two resonant frequencies $f_{1}$ and $f_{2}$ of the two orthogonal modes have been found which are equal in amplitude, so it's desired CP.

To enhance the bandwidth, suspended (central) patch has been directly coupled to two near square parasitic patches with thin resonant length of transmission line. These couplings between patches are controlled by using length and width of patches also location of the interconnecting transmission line section. This configuration is presented in Fig. 2.

The AR BW can be improved by properly choosing microstrip line length $\left(l_{s}\right)$, width $\left(W_{s}\right)$ and the size of the coupled parasitic patch. It has been observed that the reduction in microstrip line length $\left(l_{s}\right)$ (or increase in the width $W_{s}$ ) enhances the return loss BW and degrades the AR BW, this is due to the fact that the coupling between the central and parasitic patch increases. The optimized length and width of the coupling strip has been found as $8.8 \mathrm{~mm}$ and width $W_{s}=3 \mathrm{~mm}$ respectively. The bandwidth can be controlled by the length of parasitic patches $\left(l_{1}=32.8 \mathrm{~mm}\right.$ and $\left.l_{2}=32.2 \mathrm{~mm}\right)$. Figure 3 shows the optimized RL and AR plots. The optimum RL BW of $397 \mathrm{MHz}$ and AR BW of $95.16 \mathrm{MHz}$ is obtained for suspended near square central patch with two directly coupled parasitic patch antenna.

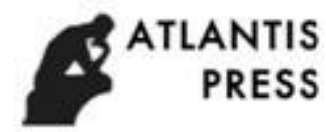




\subsection{Antenna with shorting pin}

Shorting pin position on the patch responsible for selecting operating frequency [10]. To obtain wide AR BW, antenna has been tune closer at the two AR minimums, and then a shorting pin is inserted along opposite diagonal of the parasitic patch. Fig. 3a and Fig. 3b shows the effect position of shorting pin on return loss and AR BW respectively. As pin position moves outwards along the diagonal of patch towards the corner (vertex) of patch, higher AR frequency point can be seen shifted towards right and thus offering large AR BW. When shorting pin is at point $\mathrm{P}(35.5 \mathrm{~mm}, 6.30 \mathrm{~mm})$ of parasitic patch, the AR BW of $226 \mathrm{MHz}(3.199-3.425 \mathrm{GHz})$ has been obtained.

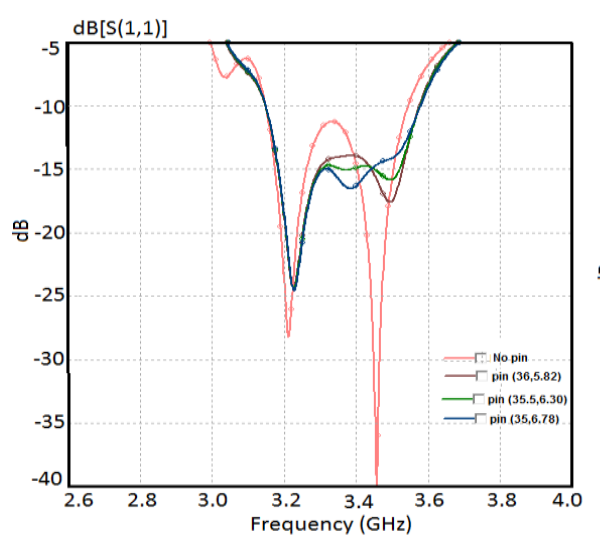

(a) Return loss plot

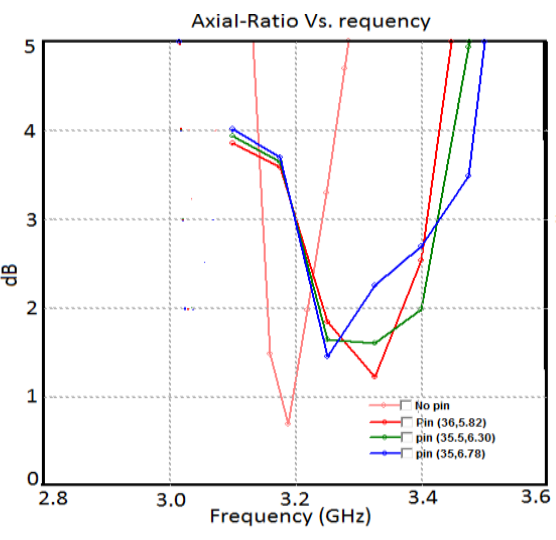

(b) AR plot

Fig. 3: Effect of shorting pin on antenna performance

Table 1. Comparison of $10 \mathrm{~dB}$ RL BW, $3 \mathrm{~dB}$ AR BW and gain with and without shorting pin

\begin{tabular}{|c|c|c|c|c|}
\hline Configuration & RL BW (MHz) & AR BW $(\mathrm{MHz})$ & $f_{r}(\mathrm{GHz})$ & Gain dBi \\
\hline Without shorting pin & $397(11.5 \%)$ & $95.16(2.98 \%)$ & 3.19 & 5.5 \\
\hline With shorting pin & $428.8(13.3 \%)$ & $226(6.79 \%)$ & 3.32 & 8.5 \\
\hline
\end{tabular}

Now let us study the effect of shorting pin on the resonant frequency and radiation directivity of directly coupled microstrip patch antenna. From the simulated results shown in Fig. 3, we can observe that antenna resonates at $3.19 \mathrm{GHz}$ without shorting pin and offers a directivity of $5.5 \mathrm{dBi}$.

As the shorting pin is loaded along the diagonal of the parasitic patch, the resonant frequency become $3.32 \mathrm{GHz}$ and the directivity become $8.5 \mathrm{dBi}$. From above analysis we can conclude that, shorting pin is responsible for shunt inductive effect and enhancement in radiation directivity is due to enlargement in electrical size of the pin loaded parasitic patch.

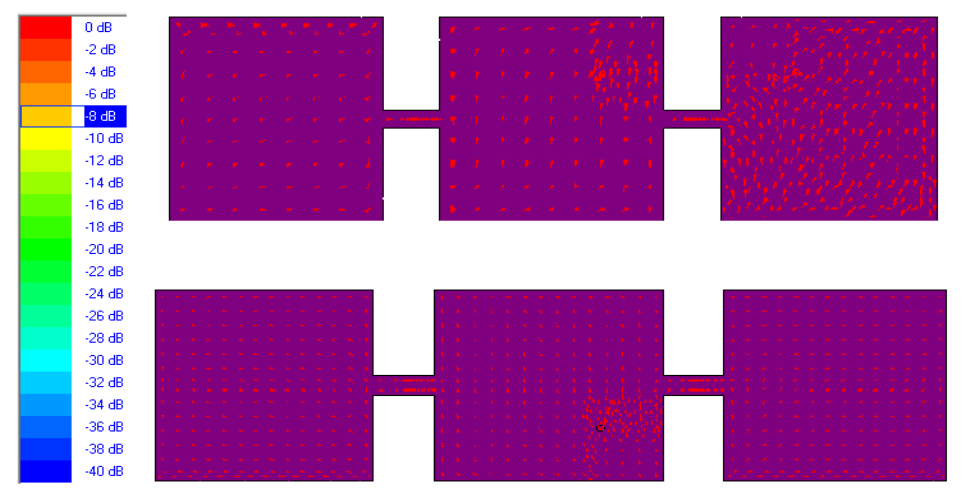

Fig. 4: Surface current distribution on patches (a) with shorting pin (3.32 GHz) and (b) without shorting pin (3.19 GHz)

Table 1 shows the comparison of $10 \mathrm{~dB}$ RL BW, AR BW and gain with and without shorting pin. Increase in resonant frequency can be clarified with the help of current distribution on patch surface shown below. 
Surface current distribution on patch with and without a shorting pin at AR frequencies is shown in Fig. 4. It can be seen that the currents of two resonant mode frequencies have increased about the shorting pin and are having equal amplitudes. Hence good CP performance has been achieved at above said frequencies. Due to shorting pin, gain of antenna is increased, which is observed from the radiation pattern of the two CP MPAs with and without shorting pins are simulated and shown in Fig. 5.

In addition, Table 2 shows the simulated gain, half-power beamwidth, radiation efficiency and directivity of the proposed antenna with and without shorting pin at their respective operating frequencies. From these table, we can compare both antenna in term of above listed parameters, antenna with shorting pin has notably narrow radiation beam (higher directivity directional gain) compared with the antenna without shorting pin. Enhancement in simulate gain of antenna is from $5.5 \mathrm{dBi}$ to $8.5 \mathrm{dBi}$ due to shorting pin. Due to enlargement in radiating area of proposed shorting pin structure, the half-power beamwidth of antenna in the two observed views/planes is significantly decreased.

Table 2. Radiation parameters of the two patches directly coupled MSA

\begin{tabular}{|c|c|c|}
\hline Antennas & Without pin & With pin \\
\hline Efficiency & $72.76 \%$ & $72.14 \%$ \\
\hline$\theta_{3 d \boldsymbol{B}}(\varphi=0)$ & 55 & 35.6 \\
\hline$\theta_{3 d \boldsymbol{B}}(\varphi=90)$ & 64 & 74 \\
\hline Directivity & $6.6 \mathrm{dBi}$ & $10.04 \mathrm{dBi}$ \\
\hline Gain & $5.5 \mathrm{dBi}$ & $8.5 \mathrm{dBi}$ \\
\hline
\end{tabular}

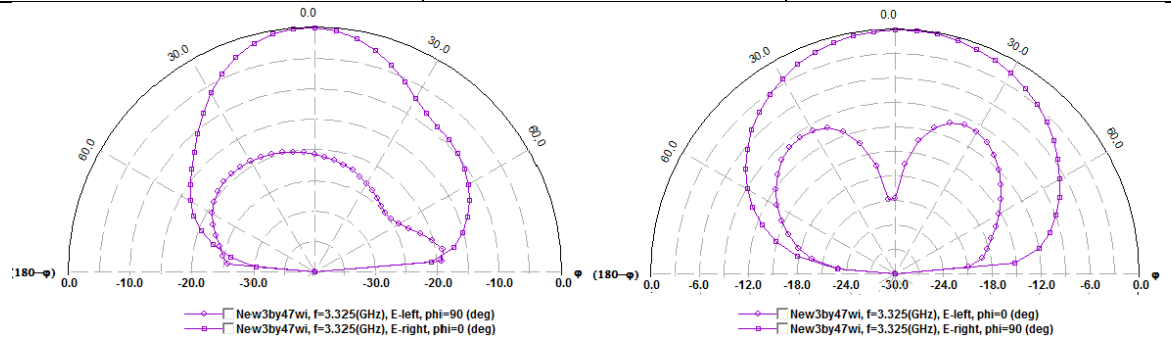

(a) With shorting pin

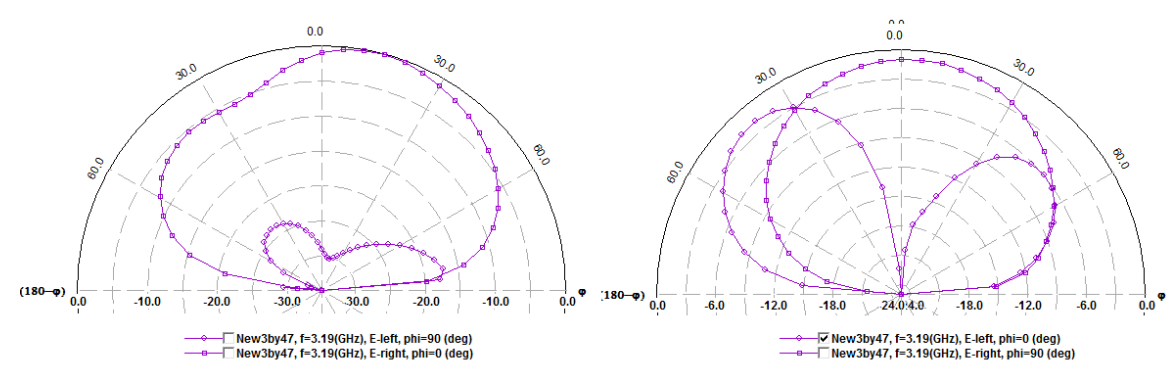

(b) Without shorting pin

Fig. 5: Radiation patterns of proposed CP MPAs

\section{Result and Discussion}

Shunt inductance effect brought by shorting pin, which is responsible for enhancement in resonant frequency and the directivity of MPA, because of increased in electrical size.

Proposed CP antenna is fabricated and measured using Agilent Technology N9923A VNA as shown in Fig. 6. Comparison of simulated and measured return loss is shown in Fig. 7. With this approach, we achieved very large measured $10 \mathrm{~dB}$ RL bandwidth of $490 \mathrm{MHz}(16.06 \%)$ with the return loss of approximately $-19.52 \mathrm{~dB}$ at $3.05 \mathrm{GHz}$, good agreement between simulated and measured value is observed as shown in Fig. 7. The proposed CP MPA yields simulated AR BW of $226 \mathrm{MHz}(6.79 \%)$ at $3.32 \mathrm{GHz}$, which we believe is a very good result as shown in Fig. 3b. Fig. 5b illustrates the simulated radiation patterns of CP MPA without pin at $3.19 \mathrm{MHz}$ and with loading pin at $3.32 \mathrm{MHz}$ in the bore-sight direction.

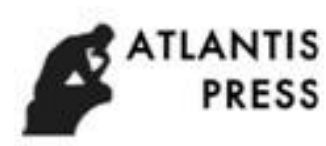


Due to radiation from the vertical feeding pins cross-polarization CP MPA with shorting pin is high for higher frequency.

These microstrip antenna configurations can be used in applications where wider AR bandwidths required. Also large cross polarization is observed over the operating frequencies as shown in Fig. 5 b.

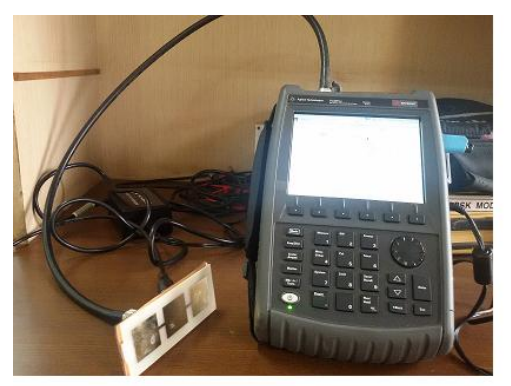

Fig. 6: Antenna testing on VNA

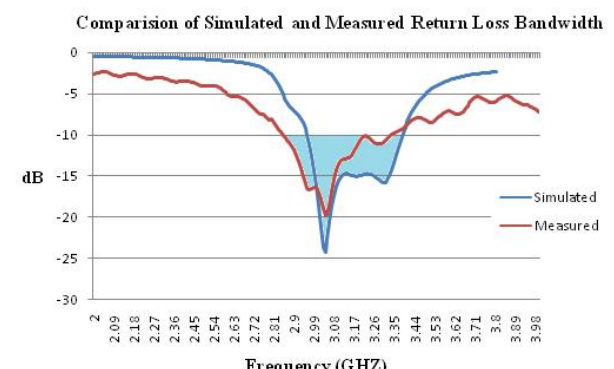

Frequency (GHZ)

\section{Conclusion}

In this paper, we presented a suspended near square patch with single feed and loaded the parasitic patch with shorting pin configuration to achieve wide AR BW and large gain. The wideband CP radiation is achieved by the excitation of the orthogonal modes in the antenna, with the shunt inductive effect of the pin, the resonant frequency of an MPA operating in its dominant mode is substantially tuned up, which enlarges the overall radiation gain and electrical radiating area of the proposed CP antenna is enhanced as a positive outcome. Finally, CP MPA with shorting pin is designed, fabricated, and tested. The simulated result of proposed antenna shows that the AR BW of the proposed CP MPA has been raised up to $6.79 \%$ from $2.98 \%$, and the gain $8.5 \mathrm{dBic}$ from 5.5 dBic. Indoor wireless communications may use the above proposed configuration where usually large AR BW is required.

\section{References}

[1] Kumar, G., Gupta, K.: Directly coupled multiple resonator wide-band microstrip antennas. IEEE Transaction on Antennas and Propagation 33(6), 588-593 (Jun 1985).

[2] Kumar, G., Ray, K.: Artech house, usa. In: Broadband Microstrip Antennas (2003).

[3] Legay, H., Shafai, L.: Series fed circularly polarised planar arrays. In: Antennas and Propagation Society International Symposium, 1994. AP-S. Digest. vol. 1, pp. 322-325 vol.1 (June 1994).

[4] Legay, H., Shafai, L.: Analysis and design of circularly polarised series fed planar subarrays. IEE Proceedings - Microwaves, Antennas and Propagation 142(2), 173- 177 (Apr 1995).

[5] R.Garg, P. Bhartia, I.b., Ittipiboon, A.: Artech house inc. In: Microstrip Antenna Design Handbook (2001).

[6] Schaubert, D., Farrar, F., Sindoris, A., Hayes, S.: Microstrip antennas with frequen cy agility and polarization diversity. IEEE Transactions on Antennas and Propagation 29(1), 118-123 (Jan 1981).

[7] Wong, K.L., Wu, J.Y.: Bandwidth enhancement of circularly-polarised microstrip antenna using chipresistor loading. Electronics Letters 33(21), 1749-1751 (Oct 1997).

[8] Wong, K.: John wiley and sons. In: A Compact and Broadband Microstrip Antennas (2002).

[9] Wu, J., Ren, X., Wang, Z., Yin, Y.: Broadband circularly polarized antenna with 1-shaped strip feeding and shorting-pin loading. IEEE Antennas and Wireless Propagation Letters 13, 1733-1736 (2014). 